\title{
ON THE MODULATION OF OSCILLATION IN THERMOHALINE CONVECTION PROBLEMS USING TEMPERATURE DEPENDENT VISCOSITY*
}

\author{
Joginder Singh Dhiman \\ Department of Mathematics, Himachal Pradesh University, \\ Summerhill, Shimla (H.P.)-171005, \\ e-mail : jsdhiman66@gmail.com \\ VIJAY KUMAR \\ Centre of Excellence, Govt. College, \\ Sanjauli, Shimla (H.P.)-171006
}

[Received 02 May 2012. Accepted 09 March 2015]

\begin{abstract}
The present paper mathematically investigates the effect of temperature dependent viscosity on the onset of instability in thermohaline convection problems of Veronis and Stern type configurations, using linear stability theory. A sufficient condition for the stability of oscillatory modes for thermohaline configuration is derived. When the compliment of this sufficient condition is true, the oscillatory motions of neutral or growing amplitude may exist, and hence the bounds for the complex growth rate of these neutral or unstable modes are derived, when viscosity of the fluid is an arbitrary function of temperature. Some general conclusions for the cases of linear and exponential variations of viscosity are worked out. The present analysis thus shows that the oscillations in thermohaline convection problems can be modulated or arrested by considering the temperature dependent viscosity of the fluid.

KEY WORDS: Thermohaline convection, viscosity variations, oscillatory motions, complex growth rate, Rayleigh number.
\end{abstract}

\section{Introduction}

Double-diffusive convection, with its archetypal case of heat and salt, generally referred to as Thermohaline convection, has been extensively studied in the recent past on account of its interesting complexities as well as its direct relevance in many problems of practical interest. Thermohaline convection has

\footnotetext{
${ }^{*}$ Corresponding author e-mail: jsdhiman66@gmail.com
} 
matured into a subject possessing fundamental departures from its classical counterpart, namely; single diffusive convection, and find applications in the fields of limnology, oceanography, geophysics, astrophysics and chemical engineering etc. The various applications of the problem have aroused the interest of many research workers, and has led to numerous research papers in various journals in the recent past. For a broad view of the subject one may be referred to Huppert \& Turner [1], Turner [2] and Brandt and Fernando [3].

Mainly, two types of fundamental configurations have been studied in the context of the thermohaline convection. First, one by Stern [4], who considered the configuration wherein the temperature gradient is stabilizing and the concentration gradient is destabilizing. He showed that a steady motion or an oscillatory motion of growing amplitude can exist for the configuration provided the destabilizing concentration gradient or the temperature gradient is sufficiently large; however, the steady motion is the preferred mode of onset of instability. On the other hand, Veronis [5] considered the case, when the temperature gradient is destabilizing and the concentration gradient is stabilizing one. In Veronis type configuration, the instability can manifests either as a stationary convection or through oscillations of growing amplitude, however the oscillatory motions of growing amplitude are the preferred mode of instability.

It is important to note here that the Veronis' work is restricted to the case of dynamically free boundaries, whereas Stern's work assumes the principle of exchange of stabilities (PES) to be valid. Since for other cases (realistic cases) of boundary combinations; i.e. when both boundaries are rigid or any combination of a rigid (on which no slip occurs) and a dynamically free (on which no tangential stress acts) boundary, the exact solutions of the eigenvalue problem are not obtainable in a closed form, and hence the values of critical Rayleigh numbers for these cases of boundary conditions are not obtainable analytically for the thermohaline convection problems. Banerjee et al. [6] proposed a noble way of combining the governing differential equation for thermohaline convection problems and consequently obtaining the bounds for positive definite integrals by exploiting the coupling between the eigen vectors. Further, Banerjee et al. [6] and Gupta et al. [7] derived the bounds for the complex growth rate of an arbitrary oscillatory perturbation for thermohaline convection problems with constant viscosity.

It is well known fact, that the viscosity of a fluid is one of the properties which are most sensitive to temperature ( $c f$. Straughan [8]). In the majority of the cases, viscosity becomes the only property which may have considerable effect on the heat transfer and the temperature variation and the dependence of other thermo-physical properties to temperature is often negligible. Torrance \& 
Turcotte [9] observed that the viscosity of the liquids decreases with increasing temperature, while the reverse trend is observed in gases. Heat transfer and pressure drop characteristics are also affected significantly with the variations in the fluid viscosity. Therefore, the type of fluid and the range of operating temperatures are very important and crucial parameters in the study of fluid dynamics, and in particular in the fields of oceanography, astrophysics etc.

In most of the studies pertaining to thermal/thermohaline convection, authors have considered the viscosity to be constant. Because of the fact that by considering the viscosity as variable (or depending upon temperature) in convective instability problems yields the eigenvalue problems in the forms of differential equations with variable coefficients, which introduces additional mathematical complexities. Some authors, including Sherman [10], Trompert and Hansen [11], Palm [12] and Stengel et al. [13]) have considered the effect of variable viscosity on the thermal convection problems. Gupta and Kaushal [14] have also analysed the stability of double-diffusive convection problems of Veronis and Stern types, by taking into account the variations in viscosity. However, the results obtained by these authors for the characterization of nonoscillatory motions for the thermohaline convection problem and the bounds for the growth rate of a neutral and unstable oscillatory perturbations are independent of viscosity variation, and hence are of limited utility.

The present paper is motivated by the above discussions and by the importance of the temperature dependence on viscosity in fluid flow problems. The main aim of the present analysis is to investigate the effect of temperature dependent viscosity on the onset of instability in thermohaline convection problems of Veronis and Stern types. In this paper, we have investigated the character of the oscillatory modes of the thermohaline convection problem and the validity of the PES in the problems, using the Pellew and Southwell [15] method. A sufficient condition for the stability of oscillatory modes in thermohaline configuration is derived. Finally, the bounds for the complex growth rate of oscillatory motions of neutral or growing amplitude of the thermohaline convection problems of both Veronis and Stern types are derived, when viscosity is an arbitrary function of temperature. Some general conclusions for the cases of linear and exponential variations of viscosity are also worked out. The obtained results are also compared with the earlier results obtained by Banerjee et al. [6], Gupta et al. [7] and Gupta and Kaushal [14] for thermohaline convection problems. 


\section{The physical configuration and eigenvalue problem}

Following the usual steps of linear stability theory and proceeding analogously as in the analyses of Gupta et al. [7] and Gupta and Kaushal [14], one can easily see that the non-dimensional linearized perturbation equations and the boundary conditions, which govern the initiation of the convection in thermohaline instability problems, when the viscosity is an arbitrary function of temperature $T$, are given by:

$$
\begin{gathered}
f\left(D^{2}-a^{2}\right)^{2} w-\frac{p}{\sigma}\left(D^{2}-a^{2}\right) w+2(D f) D\left(D^{2}-a^{2}\right) w \\
+D^{2} f\left(D^{2}+a^{2}\right) w=R_{T} a^{2} \theta-R_{S} a^{2} \phi \\
\left(D^{2}-a^{2}-p\right) \theta=-w \\
\left(D^{2}-a^{2}-\frac{p}{\tau}\right) \phi=-\frac{w}{\tau},
\end{gathered}
$$

together with either of the boundary conditions:

$$
\begin{aligned}
& \text { (4) } w=0=\theta=\phi=D^{2} w, \text { at } z=0 \text { and } z=1 \text { (Both boundaries free), } \\
& \text { (5) } w=0=\theta=\phi=D w, \text { at } z=0 \text { and } z=1 \text { (Both boundaries rigid), } \\
& \text { (6) } w=0=\theta=\phi=D w, \text { at } z=0 \text { and } w=0=\theta=\phi=D^{2} w, \text { at } z=1,
\end{aligned}
$$

(Lower boundary rigid and upper boundary dynamically free)

$$
w=0=\theta=\phi=D^{2} w, \text { at } z=0 \text { and } w=0=\theta=\phi=D w, \text { at } z=1,
$$

(Lower boundary dynamically free and upper boundary rigid).

In the foregoing equations: $w(z), \theta(z)$ and $\phi(z)$, respectively denote the perturbed velocity, temperature and concentration and are complex valued functions of vertical coordinate $z$ only; $p=p_{r}+i p_{i}$ is the growth rate; $\tau=\frac{k_{S}}{k_{T}}$ is the Lewis number with $k_{T}$ as the thermal diffusivity and $k_{S}$ as the mass diffusivity; $R_{T}=\frac{g \alpha \beta d^{4}}{k_{T} v_{0}}$ is the thermal Rayleigh number, $R_{S}=\frac{g \beta^{\prime} d^{4}}{k_{S} v_{0}}$ is the salinity Rayleigh number; $\sigma$ is Prandtl number and $a^{2}$ is the square of the wave number. Further, with $d$ as the characteristic length, the equations have 
been casted into the above dimensionless forms by using the scale factors; $\frac{z}{d}$. $k d, d \frac{d}{d z}, \frac{n d^{2}}{k_{T}}, w, \frac{k_{T} \theta}{\beta d^{2}}, \frac{v_{0}}{k_{T}}, \frac{\phi k_{S}}{\beta^{\prime} d^{2}}$ and $\frac{k_{S}}{k_{T}}$, for vertical distance, wave number, derivative $D \equiv \frac{d}{d z}$, pressure, vertical velocity, temperature, Prandtl number, concentration and Lewis number, respectively.

Also, in deriving the above equations, the viscosity of the fluid $\mu=$ $\mu_{0} f(T)$ is taken to be temperature dependent; where, $\mu_{0}$ is the viscosity at the lower boundary and $f(T)$ is any arbitrary function (which is twice continuously differentiable) of vertical coordinate $z$. Also, we have followed the assumptions of Stengel [13], regarding the small ratio of the viscosities at the top to the bottom boundaries.

System of equations (1)-(3) together with either of the boundary conditions (4)-(7) constitutes an eigenvalue problem governing the thermohaline convection problem with temperature dependent viscosity, for $R$ for given values of other parameters; namely $\sigma, R_{T}, R_{S}, \tau$ and $a^{2}$. A given state of the system is stable, neutral or unstable according as $p_{r}$ (real part of the complex growth rate $p$ ) is negative, zero or positive, respectively. Also, $p_{r} \geq 0$ and $p_{i} \neq 0$, describe the oscillatory motions of neutral or growing amplitude. Further, if $p_{r}=0$ implies $p_{i}=0$ for every wave number $a$, then the principle of exchange of stability (PES) is said to be valid, which means that instability sets in as stationary convection, otherwise we shall have overstability at least, when instability sets in as certain modes. Furthermore, the eigenvalue problem represented by equations (1)-(3) and either of the boundary conditions (4)-(7) describes:

(a) Veronis' type thermohaline convection (VTHC), when $R_{T}>0$ and $R_{S}>0 ;$ and

(b) Stern's type thermohaline convection (STHC), when $R_{T}<0$ and $R_{S}<0$.

\section{Mathematical analysis}

In this section, we shall mathematically analyze the stability of the considered problem.

(i) Stability of the oscillatory modes

We shall first investigate the character of the oscillatory modes.

Multiplying both sides of equation (1) by $w^{*}$, integrating the resulting equation by parts a suitable number of times and using the relevant boundary 
conditions (4)-(7) and equations (2)-(3), we have following equation ( $c f$. [14]);

(8) $\quad \int_{0}^{1} f\left[\left|D^{2} w\right|^{2}+2 a^{2}|D w|^{2}+a^{4}|w|^{2}\right] d z+\frac{p}{\sigma} \int_{0}^{1}\left(|D w|^{2}+a^{2}|w|^{2}\right) d z$

$$
\begin{gathered}
+a^{2} \int_{0}^{1} D^{2} f|w|^{2} d z=R_{T} a^{2} \int_{0}^{1}\left[|D \theta|^{2}+a^{2}|\theta|^{2}\right] d z \\
-R_{S} a^{2} \tau \int_{0}^{1}\left[|D \phi|^{2}+a^{2}|\phi|^{2}\right] d z+a^{2} p^{*}\left[R_{T} \int_{0}^{1}|\theta|^{2} d z-R_{S} \int_{0}^{1}|\phi|^{2} d z\right] .
\end{gathered}
$$

Comparing the real and imaginary parts of both sides of equation (8), we have:

$$
\begin{aligned}
& \int_{0}^{1} f\left|D^{2} w\right|^{2} d z+2 a^{2} \int_{0}^{1} f\left(|D w|^{2}+a^{4}|w|^{2}\right) d z \\
& +\frac{p_{r}}{\sigma} \int_{0}^{1}\left(|D w|^{2}+a^{2}|w|^{2}\right) d z+a^{2} \int_{0}^{1} D^{2} f|w|^{2} d z \\
& -R_{T} a^{2} \int_{0}^{1}\left[|D \theta|^{2}+a^{2}|\theta|^{2}\right] d z+R_{S} a^{2} \int_{0}^{1} \tau\left[|D \phi|^{2}+a^{2}|\phi|^{2}\right] d z \\
& \quad+a^{2} p_{r}\left(R_{T} \int_{0}^{1}\left[|\theta|^{2} d z-R_{S} \int_{0}^{1}|\phi|^{2} d z\right] d z\right)=0
\end{aligned}
$$

and

$$
p_{i}\left[\int_{0}^{1}\left(|D w|^{2}+a^{2}|w|^{2}\right) d z+R_{T} a^{2} \sigma \int_{0}^{1}|\theta|^{2} d z-R_{S} a^{2} \sigma \int_{0}^{1}|\phi|^{2} d z\right]=0
$$

It is clear from equation (10), that $p_{i}$ may or may not be zero, which in view of above discussions means that PES in general is not valid for both VTHC and STHC problems and thus, instability can also arise through oscillations.

Let us suppose, that $p_{i} \neq 0$, i.e. PES is not valid. This fact means that the instability is through oscillation. So, cancelling $p_{i}$ throughout from equation (10), we can have:

$$
\int_{0}^{1}\left(|D w|^{2}+a^{2}|w|^{2}\right) d z+R_{T} a^{2} \sigma \int_{0}^{1}|\theta|^{2} d z-R_{S} a^{2} \sigma \int_{0}^{1}|\phi|^{2} d z=0
$$

which yields, that:

$$
R_{S} a^{2} \int_{0}^{1}|\phi|^{2} d z>\frac{1}{\sigma} \int_{0}^{1}\left(|D w|^{2}+a^{2}|w|^{2}\right) d z
$$


On the Modulation of Oscillation in Thermohaline Convection Problems ...45

Since $w, \theta$ and $\phi$ satisfy the boundary conditions:

$$
w(0)=0=w(1) ; \theta(0)=0=\theta(1) ; \text { and } \phi(0)=0=\phi(1),
$$

therefore, upon using the Rayleigh-Ritz inequality [16], we have:

$$
\begin{aligned}
& \int_{0}^{1}|D w|^{2} d z \geq \pi^{2} \int_{0}^{1}|w|^{2} d z, \\
& \int_{0}^{1}|D \theta|^{2} d z \geq \pi^{2} \int_{0}^{1}|\theta|^{2} d z \\
& \int_{0}^{1}|D \phi|^{2} d z \geq \pi^{2} \int_{0}^{1}|\phi|^{2} d z .
\end{aligned}
$$

Further:

$$
\begin{gathered}
\int_{0}^{1}\left(\left.D \theta\right|^{2}+a^{2}|\theta|^{2}\right) d z=\left|-\int_{0}^{1} \theta^{*}\left(D^{2}-a^{2}\right) \theta d z\right| \leq\left|\int_{0}^{1} \theta^{*}\left(D^{2}-a^{2}\right) \theta d z\right| \\
\leq\left|\int_{0}^{1}\right| \theta||\left(D^{2}-a^{2}\right) \theta|d z| \leq\left[\int_{0}^{1}|\theta|^{2} d z\right]^{\frac{1}{2}}\left[\int_{0}^{1}\left|\left(D^{2}-a^{2}\right) \theta\right|^{2} d z\right]^{\frac{1}{2}},
\end{gathered}
$$

(Using Schwartz Inequality).

Also,

$$
\begin{array}{r}
\int_{0}^{1}\left(|D \theta|^{2}\right) d z=\left|-\int_{0}^{1} \theta^{*} D^{2} \theta d z\right| \leq\left|\int_{0}^{1} \theta^{*} D^{2} \theta d z\right| \leq\left.\left|\int_{0}^{1}\right| \theta\right|^{2}\left|D^{2} \theta\right|^{2} d z \mid \\
\leq\left[\int_{0}^{1}|\theta|^{2} d z\right]^{\frac{1}{2}}\left[\int_{0}^{1}\left|D^{2} \theta\right|^{2} d z\right]^{\frac{1}{2}},
\end{array}
$$

(Using Schwartz Inequality).

Using inequality (13), inequality (16) implies that:

$$
\int_{0}^{1}\left|D^{2} \theta\right|^{2} d z \geq \pi^{4} \int_{0}^{1}|\theta|^{2} d z .
$$

Also, proceeding analogously as in the derivation of the inequality (17), we can have:

$$
\int_{0}^{1}\left|D^{2} w\right|^{2} d z \geq \pi^{4} \int_{0}^{1}|w|^{2} d z .
$$


Combining inequalities (12) and (18), we can get:

$$
\int_{0}^{1} f\left[\left|D^{2} w\right|^{2}+2 a^{2}|D w|^{2}+a^{4}|w|^{2}\right] d z \geq f_{\min }\left(\pi^{2}+a^{2}\right)^{2} \int_{0}^{1}|w|^{2} d z
$$

where, $f_{\min }$ is the minimum value of $f(z)$ in the closed interval $[0,1]$.

Further, multiplying equation (2) by its complex conjugate and integrating the various terms on the left hand side of the resulting equation by parts for an appropriate number of times and making use of boundary conditions: $\theta(0)=\theta(1)=0$, we get:

$$
\begin{aligned}
\int_{0}^{1}\left[\left|D^{2} \theta\right|^{2}+2 a^{2}|D \theta|^{2}+a^{4}|\theta|^{2}\right] d z+2 p_{r} & \int_{0}^{1}\left(|D \theta|^{2}+a^{2}|\theta|^{2}\right) \\
& +|p|^{2} \int_{0}^{1}|\theta|^{2} d z=\int_{0}^{1}|w|^{2} d z .
\end{aligned}
$$

If permissible, let $p_{r} \geq 0$. In view of this fact, equation (20) implies that:

$$
\int_{0}^{1}\left|\left(D^{2}-a^{2}\right) \theta\right|^{2} d z=\int_{0}^{1}\left[\left|D^{2} \theta\right|^{2}+2 a|D \theta|^{2}+a^{4}|\theta|^{2}\right] d z<\int_{0}^{1}|w|^{2} d z .
$$

Further, combining inequalities (13) and (17), we have:

$$
\int_{0}^{1}\left[\left|D^{2} \theta\right|^{2}+2 a^{2}|D \theta|^{2}+a^{4}|\theta|^{2}\right] d z \geq\left(\pi^{2}+a^{2}\right)^{2} \int_{0}^{1}|\theta|^{2} d z .
$$

Inequalities (15), upon using inequalities (21) and (22), yields:

$$
\int_{0}^{1}\left[|D \theta|^{2}+a^{2}|\theta|^{2} d z<\frac{1}{\left(\pi^{2}+a^{2}\right)} \int_{0}^{1}|w|^{2} d z .\right.
$$

We can also derive the following inequalities analogous to the derivation of inequality (22):

$$
\begin{gathered}
\int_{0}^{1}\left[|D w|^{2}+a^{2}|w|^{2}\right] d z \geq\left(\pi^{2}+a^{2}\right) \int_{0}^{1}|w|^{2} d z \\
\int_{0}^{1}\left[|D \phi|^{2}+a^{2}|\phi|^{2}\right] d z \geq\left(\pi^{2}+a^{2}\right) \int_{0}^{1}|\phi|^{2} d z .
\end{gathered}
$$


Now, multiplying equation (10) by $p_{r}$ and adding the resulting equation to equation (9), we have:

$$
\begin{gathered}
\int_{0}^{1} f\left[\left|D^{2} w\right|^{2}+2 a^{2}|D w|^{2}+a^{4}|w|^{2}\right] d z+2 \frac{p_{r}}{\sigma} \int_{0}^{1}\left(|D w|^{2}+a^{2}|w|^{2}\right) d z \\
-R_{T} a^{2} \int_{0}^{1}\left[|D \theta|^{2}+a^{2}|\theta|^{2}\right] d z+a^{2} \int_{0}^{1} D^{2} f|w|^{2} d z \\
+R_{S} a^{2} \tau \int_{0}^{1}\left[|D \phi|^{2}+a^{2}|\phi|^{2}\right] d z=0 .
\end{gathered}
$$

For $D^{2} f \geq 0$ (double derivative of the function $f$ greater than or equal to zero), equation (26) with $p_{r} \geq 0$, yields:

$$
\begin{aligned}
\int_{0}^{1} f\left[\left|D^{2} w\right|^{2}+2 a^{2}|D w|^{2}+a^{4}|w|^{2}\right] d z+ & R_{S} a^{2} \tau \int_{0}^{1}\left[|D \phi|^{2}+a^{2}|\phi|^{2}\right] d z \\
& <R_{T} a^{2} \int_{0}^{1}\left[|D \theta|^{2}+a^{2}|\theta|^{2}\right] d z .
\end{aligned}
$$

Also, inequality (11), upon using inequality (25), yields:

$$
R_{S} a^{2} \int_{0}^{1}|\phi|^{2} d z>\frac{\left(\pi^{2}+a^{2}\right)}{\sigma} \int_{0}^{1}|w|^{2} d z .
$$

Now using inequalities (19), (23) and (28) in inequality (27), we have:

$$
\frac{\left(\pi^{2}+a^{2}\right)^{3}}{a^{2}}\left(f_{\min }+\frac{\tau}{\sigma}\right) \int_{0}^{1}|w|^{2} d z<R_{T} \int_{0}^{1}|w|^{2} d z .
$$

Since, minimum value of $\frac{\left(\pi^{2}+a^{2}\right)^{3}}{a^{2}}$ with respect to $a^{2}$ is $\frac{27 \pi^{4}}{4}$, therefore inequality (29) yields:

$$
\left[\frac{27 \pi^{4}}{4}\left(f_{\min }+\frac{\tau}{\sigma}\right)-R_{T}\right] \int_{0}^{1}|w|^{2} d z<0 .
$$

From the above inequality, it is clear that if $R_{T}<\frac{27 \pi^{4}}{4}\left(f_{\min }+\frac{\tau}{\sigma}\right)$, we have a contradiction. Hence, we must have $p_{r}<0$.

The above result clearly implies that the oscillatory modes of the system are stable, when $D^{2} f \geq 0$ and $R_{T}<\frac{27 \pi^{4}}{4}\left(f_{\min }+\frac{\tau}{\sigma}\right)$. Further, proceeding exactly as in the above analysis, replacing $R_{T}$ and $R_{s}$, respectively 
with $-\left|R_{T}\right|$ and $-\left|R_{s}\right|$ and following the analogous steps, we have a sufficient condition for the stability of the oscillatory modes for STHC problem as: $\left|R_{S}\right|<\frac{27 \pi^{4} \tau}{4}\left(f_{\min }+\frac{1}{\sigma}\right)$ with $D^{2} f \geq 0$.

It is remarkable to note, that when we consider the compliment of the above sufficient condition for the stability of the oscillatory motions, i.e. when $R_{T} \geq \frac{27 \pi^{4}}{4}\left(f_{\min }+\frac{\tau}{\sigma}\right)$, the oscillatory modes of growing amplitude may exist. Hence, it becomes important to prescribe the bounds for the growth rate of these motions.

In the following analysis, we have derived such bounds, which arrest the complex growth rate of the arbitrary neutral or unstable $\left(p_{r} \geq 0\right)$ oscillatory motions $\left(p_{i} \neq 0\right)$.

(ii) Bounds for complex growth rate for VTHC

Taking $p_{r} \geq 0$ in equation (21) and using inequality (22) in the resulting inequality, we get the following inequality:

$$
\int_{0}^{1}|\theta|^{2} d z<\frac{1}{\left(\pi^{2}+a^{2}\right)^{2}+|p|^{2}} \int_{0}^{1}|w|^{2} d z .
$$

Now, using inequalities (30) and (21) in inequality (15), we have:

$$
\int_{0}^{1}\left[|D \theta|^{2}+a^{2}|\theta|^{2}\right] d z<\frac{1}{\left(\pi^{2}+a^{2}\right)\left[1+\frac{|p|^{2}}{\left(\pi^{2}+a^{2}\right)^{2}}\right]^{\frac{1}{2}}} \int_{0}^{1}|w|^{2} d z .
$$

So, using inequalities (19), (28) and (31), inequality (27) yields the following inequality:

$$
\left(\frac{\pi^{2}+a^{2}}{a^{2}}\right)^{3}\left(f_{\min }+\frac{\tau}{\sigma}\right) \int_{0}^{1}|w|^{2} d z<\frac{R_{T}}{\left[1+\frac{|p|^{2}}{\left(\pi^{2}+a^{2}\right)^{2}}\right]^{\frac{1}{2}}} \int_{0}^{1}|w|^{2} d z
$$

Since, minimum value of $\frac{\left(\pi^{2}+a^{2}\right)^{3}}{a^{2}}$ with respect to $a^{2}$ is $\frac{27 \pi^{4}}{4}$, therefore inequality (32) yields:

$$
\left[\frac{27 \pi^{4}}{4}\left(f_{\min }+\frac{\tau}{\sigma}\right)-\frac{R_{T}}{\left[1+\frac{|p|^{2}}{\left(\pi^{2}+a^{2}\right)^{2}}\right]^{\frac{1}{2}}}\right] \int_{0}^{1}|w|^{2} d z<0 .
$$



implies that:

Inequality (33), under the condition $R_{T} \geq \frac{27 \pi^{4}}{4}\left(f_{\min }+\frac{\tau}{\sigma}\right)$ clearly

$$
|p|<\left(\pi^{2}+a^{2}\right) \sqrt{\left(Q^{2}-1\right)},
$$

where, $Q=\frac{4 \sigma R_{T}}{27 \pi^{4}\left(\tau+\sigma \cdot f_{\min }\right)}$.

Also, from inequality (32), we can have:

$$
\left(\pi^{2}+a^{2}\right)\left(\frac{\pi^{2}+a^{2}}{a^{2}}\right)^{2}\left(f_{\min }+\frac{\tau}{\sigma}\right)<R_{T} .
$$

Because, minimum value of $\frac{\left(\pi^{2}+a^{2}\right)^{2}}{a^{2}}$ with respect to $a^{2}$ is $4 \pi^{2}$, therefore inequality (35) yields:

$$
\left(\pi^{2}+a^{2}\right)<\frac{1}{4 \pi^{2}}\left(\frac{\sigma R_{T}}{\tau+\sigma f_{\min }}\right) .
$$

Now, using inequality (36) in inequality (34), we get:

$$
|p|<\frac{1}{4 \pi^{2}}\left(\frac{\sigma R_{T}}{\tau+\sigma f_{\min }}\right) \sqrt{\left(Q^{2}-1\right)} .
$$

The above result can be stated in the following theorem:

Theorem 1. If $(p, w, \theta, \phi), p=p_{r}+i p_{i}, p_{r} \geq 0, p_{i} \neq 0, R_{T}>0$ and $R_{S}>0, D^{2} f \geq 0, R_{T}>\frac{27 \pi^{4}}{4}\left(f_{\min }+\frac{\tau}{\sigma}\right)$ is a solution of equations (1)-(3) together with either of boundary conditions (4)-(7), then:

$$
|p|<\frac{\sigma R_{T}}{4 \pi^{2}\left(\tau+\sigma f_{\min }\right)} \sqrt{\left(Q^{2}-1\right)}
$$

where: $Q=\frac{4 \sigma R_{T}}{27 \pi^{4}\left(\tau+\sigma \cdot f_{\min }\right)}$.

In the terminology of the hydrodynamic stability theory, the above result can be stated as:

"The complex growth rate $p=p_{r}+i p_{i}$ of an arbitrary oscillatory $\left(p_{i} \neq 0\right)$ perturbation of growing amplitude $\left(p_{r} \geq 0\right)$, in a Veronis' Type thermohaline convection with temperature dependent viscosity when $D^{2} f \geq 0$, lies inside a semicircle in the right half of the $p_{r} p_{i}$-plane, whose centre is at the origin and whose radius is $\frac{1}{4 \pi^{2}}\left(\frac{\sigma R_{T}}{\tau+\sigma f_{\min }}\right) \sqrt{\left(Q^{2}-1\right)}$. Further, the result is uniformly valid for the all combinations of rigid and dynamically free boundary conditions. 
(iii) Bounds for complex growth rate for STHC

Following the analysis adopted in the derivation of the results for the case of VTHC problem, analogous results can be easily derived for the case of STHC problem, just by replacing $R_{T}$ and $R_{S}$ with $-\left|R_{T}\right|$ and $-\left|R_{S}\right|$ and following the analogous steps. Thus, the bound for the complex growth rate for STHC with temperature dependent viscosity is given by:

Theorem 2. If $(p, w, \theta, \phi), p=p_{r}+i p_{i}, p_{r} \geq 0, p_{i} \neq 0, D^{2} f \geq 0$, $R_{T}<0$ and $R_{S}<0,\left|R_{S}\right|>\frac{27 \pi^{4} \tau}{4}\left(f_{\min }+\frac{1}{\sigma}\right)$ is a solution of equations (1)-(3) together with either of boundary conditions (4)-(7), then:

$$
|p|<\frac{\sigma\left|R_{S}\right|}{4 \pi^{2}\left(1+\sigma f_{\min }\right)} \sqrt{\left(\tilde{Q}^{2}-1\right)}
$$

where: $\tilde{Q}=\frac{4 \sigma\left|R_{S}\right|}{27 \pi^{4} \tau\left(1+\sigma \cdot f_{\min }\right)}$.

The essential contents of the Theorem 2, from the point of view of hydrodynamic stability theory are similar to those of Theorem 1and the remarks drawn for the case of VTHC are analogously valid for the present case of STHC, also.

\section{Conclusion and discussion}

The above analysis establishes that the oscillatory modes of growing amplitude for Veronis' Type thermohaline convection problem with variable viscosity are not allowed, if the thermal Rayleigh number $R_{T}<\frac{27 \pi^{4}}{4}\left(f_{\min }+\frac{\tau}{\sigma}\right)$ together with the second derivative of the viscosity function is positive or zero $\left(D^{2} f \geq 0\right)$ which clearly shows that the stability of the oscillatory motions depends upon the viscosity of the fluid.

In particular, when the viscosity is varying linearly or exponentially, the condition $D^{2} f \geq 0$ on viscosity is automatically satisfied and $f_{\min }=1$, for both cases of viscosity variations. In view of the above facts, the above sufficient condition yields $R_{T}<\frac{27 \pi^{4}}{4}\left(1+\frac{\tau}{\sigma}\right)$, which is the same condition as obtained by Gupta et al. [7] for Veronis' Type thermohaline convection problem with constant viscosity. Thus, from the present analysis, we observe that whether the viscosity is constant or varying linearly and exponentially, the sufficient condition for the stability of oscillatory modes is $R_{T}<\frac{27 \pi^{4}}{4}\left(1+\frac{\tau}{\sigma}\right)$. The obtained result thus generalizes the results of Gupta et. al. [7] to the case of 
viscosity variation. Also, the above remarks for VTHC are also analogously valid for the stability of the oscillatory modes for STHC problem also.

As stated earlier, if the compliment of the above conditions for the stability of the oscillatory modes of VTHC and STHC problems are true, we may have instability through oscillations. Hence, Theorems 1 and 2 provides the bounds for the growth of complex rates of oscillatory motions of neutral or growing amplitude. We observed from Theorem 1 that the radius of the growth rate for these oscillatory perturbations clearly depends upon the viscosity of the fluid and hence by modulating the viscosity through temperature, one can control the growth rate. Theorem 1 also yields that when $Q<1$, i.e. $R_{T}<$ $\frac{27 \pi^{4}}{4}\left(f_{\min }+\frac{\tau}{\sigma}\right)$, the oscillatory motions of growing amplitude are not allowed, which is the same condition as derived above. Further, when the viscosity is varying linearly or exponentially, we have $D^{2} f \geq 0$ and $f_{\min }=1$, the bound obtained in Theorem 1 reduces to

$$
|p|<\frac{\sigma R_{T}}{4 \pi^{2}(\tau+\sigma)} \sqrt{Q^{2}-1}, \text { where } Q=\frac{4 \sigma R_{T}}{27 \pi^{4}(\tau+\sigma)},
$$

which is the same bound as obtained by Gupta et al. [7] for VTHC problem with constant viscosity. Thus, the above remarks clearly establish the generality of the results derived herein with temperature dependent viscosity.

Further, from the expression given in Theorem 1, we can see that the radius of the arbitrary oscillatory perturbation decreases as the minimum value of $f(T)$ increases, which means that more the viscosity of the fluid smaller is the region for the growth rate of oscillatory perturbation. Hence, we concluded that by modulating the viscosity of the fluid with temperature, one can control the oscillation in thermohaline convection.

The essential contents of Theorem 2 from the point of view of hydrodynamic stability theory are similar to those of Theorem 1 . Hence, the conclusions and remarks drawn for the case of VTHC are analogously valid for the present case of STHC. Further, the above derived results are uniformly valid for the quite general nature of boundaries and hence are of wider applicability.

\section{REFERENCES}

[1] Huppert, H. E., J. S. Turner. Double-diffusive Convection. J. Fluid Mech., 106 (1981), 299-329.

[2] Turner, J. S. Convection in Multicomponent Systems. Naturwissenschaften, 72 (1985), No. 2, 70-75. 
[3] Brandt, A., H. J. S. Fernando. Double Diffusive Convection, DC, Washington, Am. Geophys. Union, 1996.

[4] Stern, M. E. The Salt Fountain and Thermohaline Convection. Tellus, 12 (1960), 172-175.

[5] Veronis, G. On Finite Amplitude Instability in Thermohaline Convection. J. Mar. Res., 23 (1965), No. 1, 1-17.

[6] Banerjee, M. B., D. C. Katoch, G. S. Dube, K. Banerjee. Bounds for Growth Rate of Perturbation in Thermohaline Convection. Proc. Roy. Soc., London, A387 (1981), 301-304.

[7] Gupta, J. R., J. S. Dhiman, J. Thakur. Thermohaline Convection of Veronis and Stern Types Revisited. J. Math. Anal. Appl., 264 (2001), 398-407.

[8] Straughan, B. Sharp Global Non-linear Stability for Temperature Dependent Viscosity. Proc. R. Soc., London, A458 (2002), 1773-1782.

[9] Torrance, K. E., D. L. Turcotte. Thermal Convection with Large Viscosity Variations. J. Fluid Mech., 47 (1971), 113-125.

[10] Sherman, F. S. Viscous Flow, NY, McGraw Hill Book Co., 1990.

[11] Trompert, R. A., U. Hansen. On the Rayleigh Number Dependence of Convection with a Strongly Temperature Dependent Viscosity. Phys. Fluids, 10 (1998), No. 2, 351-360.

[12] Palm, E. On the Tendency Towards Hexagonal Calls in Steady Convection. J. Fluid Mech., 8 (1960), 183-192.

[13] Stengel, K. C., D. S. Oliver, J. R. Booker. Onset of Convection in a Variable Viscosity Fluid. J. Fluid Mech., 120 (1982), 411-431.

[14] Gupta, J. R., M. B. Kaushal. Rotatory Hydromagnetic Double- diffusive Convection with Viscosity Variations. J. Math. Phys. Sci., 22 (1988), No. 3, 301-320.

[15] Pellew, A., R. V. Southwell. On Maintained Convective Motion in a Fluid Heated from Below. Proc. Roy. Soc. London A, 176 (1940), 312-343.

[16] Schultz, M. H. Spline Analysis, NJ, Prentice-Hall, Englewood Cliffs, 1973. 